\title{
VALUE DISTRIBUTION OF POTENTIALS IN THREE REAL VARIABLES ${ }^{1}$ \\ PETER A. MCCOY
}

\begin{abstract}
The object of this paper is to study the value distribution of potentials in three real variables by means of the Bergman integral operator with methods drawn from the analytic theory of polynomials.
\end{abstract}

In this paper we obtain results on the value distribution of nonaxisymmetric potentials which generalize recent papers of the author [3] and of Morris Marden [2] which treat the value distribution of axisymmetric potentials, generalized axisymmetric potentials, and in Marden's paper, some special classes of harmonic polynomials in three real variables.

Let $(x, y, z)$ be a point in $E^{3}$. A function $\Psi=\Psi(x, y, z)$ with continuous second partial derivatives in a domain $G$ shall be referred to as a potential if it satisfies

$$
\Psi_{x x}+\Psi_{y y}+\Psi_{z z}=0
$$

in $G$.

Bergman has shown [1, p. 43] that potentials are generated by the integral operator

$$
\Psi(x, y, z)=\frac{1}{2 \pi i} \int_{\mathscr{L}} f(\tau, \zeta) \zeta^{-1} d \zeta
$$

where the function $f(\tau, \zeta)$ which is the associate of $\Psi$ is an analytic function of $\tau$ for all $\tau$ over some region $\Omega$ in the complex plane and continuous in $\zeta$, for $\zeta$ over the contour $\mathscr{L}$ with

$$
\tau=x+\frac{1}{2}(i y+z) \zeta+\frac{1}{2}(i y-z) \zeta^{-1} .
$$

The Bergman operator (2) shall be designated by

$$
\Psi(x, y, z)=B(f, \mathscr{L}, \boldsymbol{X}), \quad \boldsymbol{X}=(x, y, z) .
$$

Presented to the Society, June 17, 1972; received by the editors February 7, 1972 and, in revised form, May 8, 1972.

AMS (MOS) subject classifications (1970). Primary 31B05, 44A15; Secondary 30A08, 30A70.

1 This paper is based on a part of the author's Ph.D. dissertation which was directed by Professor Morris Marden at the University of Wisconsin-Milwaukee.

(c) American Mathematical Society 1973 
The contour $\mathscr{L}$ shall be referred to as an admissible contour if it is a simple smooth contour which is homologous to the unit circle centered at the origin mod the singularities of the integrand considered as a function of $\zeta$. To facilitate the statements of our results we consider the following definitions.

Let $\alpha$ and $\beta$ be real constants where $0<\alpha \leqq \pi / 2$. The sector defined by

$$
\beta-\alpha<\arg \zeta<\alpha+\beta
$$

shall be denoted by $s(\alpha, \beta)$.

An admissible contour $\mathscr{L}$ which is a simple closed contour and has a parametric representation of the form $a=a(t)$ for $0 \leqq t \leqq 1$ where $a^{-1} a^{\prime}$ maps $[0,1]$ into $s(\alpha, \beta)$ shall be referred to as a contour $\mathscr{L}$ of type $s(\alpha, \beta)$. Let $p_{0}=\left(x_{0}, y_{0}, z_{0}\right)$ and let $\gamma$ and $\delta$ be real constants such that the point

$$
F(\zeta)=f\left(x_{0}+\frac{1}{2}\left(i y_{0}+z_{0}\right) \zeta+\frac{1}{2}\left(i y_{0}-z_{0}\right) \zeta^{-1}, \zeta\right)
$$

is contained in $s(\gamma, \delta)$ for all $\zeta$ on a contour $\mathscr{L}$ of type $s(\alpha, \beta)$. The maximal open arcwise connected set in $E^{3}$ containing $p_{0}$ such that for each point $(x, y, z)$ that it contains, $f(\tau, \zeta)$ is defined, single valued, and in $s(\gamma, \delta)$ for all $\zeta$ on $\mathscr{L}$, shall be referred to as a basic region determined by $p_{0}$. This basic region shall be represented by $\Gamma\left(f, p_{0}, \gamma, \delta\right)$.

Let $\varepsilon$ be a real nonzero number with sufficiently small absolute value. Two basic regions determined by $p_{0}, \Gamma\left(f, p_{0}, \gamma, \delta\right)$ and $\Gamma\left(f, p_{0}, \gamma, \delta+\varepsilon\right)$, shall be called continuations of each other if neither is a subset of the other, and if their intersection is nonvoid. An example of a basic region which has no continuation follows: Let

$$
f(\tau, \zeta)=\exp (x+i y \cos t+i z \sin t) \text { with } 0 \leqq t \leqq 2 \pi .
$$

This function may be represented in cylindrical coordinates $(x, \rho, \varphi)$ with

$$
y^{2}+z^{2}=\rho^{2}, \quad y=\rho \cos \varphi, \quad z=\rho \sin \varphi
$$

as $\Psi(p, t)=\exp (x+i \rho \cos (t-\varphi))$ with $p=(x, \rho, \varphi)$.

Let $q$ be the origin in $E^{3}$ and $\Gamma(\pi / 2,0)=\Gamma(\Psi, q, \pi / 2,0)$ be a basic region determined by $q$. It is immediate that

$$
\Gamma(\pi / 2,0)=\{(x, \rho, p) \mid 0 \leqq \rho<\pi / 2\} .
$$

Assume that $\Gamma(\pi / 2,0)$ has a continuation $\Gamma(\pi / 2, \varepsilon)=\Gamma(\Psi, q, \pi / 2, \varepsilon)$. Then $\Gamma(\pi / 2, \varepsilon)-\Gamma(\pi / 2,0)$ contains a point of the form

$$
p_{1}=\left(x_{1}, \pi / 2+\delta_{1}, \varphi_{1}\right)
$$

with $\delta_{1}>0$. There exist points $t_{0}$ and $t_{1}$ in $[0,2 \pi]$ such that $\arg \Psi\left(p_{1}, t_{0}\right)=$ $-\pi / 2-\delta_{1}$ and such that $\arg \Psi\left(p_{1}, t_{1}\right)=\pi / 2+\delta_{1}$. But, since $p_{1}$ is contained 
in $\Gamma(\pi / 2, \varepsilon), \varepsilon-\pi / 2<\arg \Psi\left(p_{1}, t\right)<\varepsilon+\pi / 2$ for $0 \leqq t \leqq 2 \pi$ which is impossible. Thus, $\Gamma(\pi / 2,0)$ has no continuation.

We shall require the additional definitions. The totality of all continuations of a basic region determined by $p_{0}$ shall be designated by $E\left(f, p_{0}, \gamma\right)$ and referred to as the region associated with $p_{0}$. A region associated with a point $p$ determines an equivalence class of points in $E^{3}$ in an obvious manner. For fixed $\gamma$, the set obtained from the union of all such equivalence classes $E(f, p, \gamma)$ shall be designated by $\Omega(f, \gamma)$ and referred to as a fundamental region of $f$.

THEOREM. Let $\mu$ and $\lambda$ be real positive numbers whose sum is $\pi / 2$ and $\mathscr{L}_{0}$ be a contour of type $s(\mu, \gamma)$. Let $\Psi(x, y, z)$ be the potential generated by $B\left(f, \mathscr{L}_{0}, X\right)$ where the function $f(\tau, \zeta)$ is analytic in $\tau$ for $\tau$ over a region $\Omega$ in the complex plane and continuous in $\zeta$ for all $\zeta$ on $\mathscr{L}_{0}$. Then, for any complex constant $\eta$ for which $\Omega(f-\eta, \lambda)$ is nonvoid,

$$
\Psi(x, y, z) \neq \eta n\left(\mathscr{L}_{0}, 0\right) \quad \text { where } \quad n\left(\mathscr{L}_{0}, 0\right)=\frac{1}{2 \pi i} \int_{\mathscr{L}_{0}} \zeta^{-1} d \zeta .
$$

Proof. Choose any point $(x, y, z)$ in $\Omega(f-\eta, \lambda)$. Such a point necessarily lies in a region $E\left(f-\eta, p_{1}, \lambda\right)$, associated with some point $p_{1}$, and thus in some basic region $\Gamma\left(f-\eta, p_{1}, \lambda, \omega\right)$ associated with $p_{1}$, for some $\omega$ in $[0,2 \pi]$. This containment implies that

$$
\omega-\lambda<\arg (f(\tau, \zeta)-\eta)<\omega+\lambda
$$

for all $\zeta$ on $\mathscr{L}_{0}$. As the contour $\mathscr{L}_{0}$ is of type $s(\mu, \gamma)$, it has a parametric representation of the form $\zeta=\zeta(t)$ for $0 \leqq t \leqq 1$ where

$$
\gamma-\mu<\arg \left(\zeta^{-1}(t) \zeta^{\prime}(t)\right)<\gamma+\mu
$$

for $0 \leqq t \leqq 1$. From inequalities (7) and (8) we find that, for each $t$ on $0 \leqq t \leqq 1$,

(9) $\gamma+\omega-\lambda-\mu<\arg \left\{(f(\tau, \zeta)-\eta) \zeta^{-1}(t) \zeta^{\prime}(t)\right\}<\lambda+\mu+\gamma+\omega$.

By considering the integrand of

$$
\Psi(x, y, z)-\eta n\left(\mathscr{L}_{0}, 0\right)=\frac{1}{2 \pi i} \int_{\mathscr{L}_{0}}(f(\tau, \zeta)-\eta) \zeta^{-1} d \zeta
$$

as the limit of a sum of vectors whose sum by inequality (9) terminates in the open half plane $s(\pi / 2, \gamma+\omega)$, we find that the integral cannot vanish. Thus in $\Omega(f-\eta, \lambda)$,

$$
\Psi(x, y, z) \neq \eta n\left(\mathscr{L}_{0}, 0\right)
$$

as was to be proved. We note that for any $\lambda, 0<\lambda \leqq \pi / 2$, there exists a complex constant $\eta$ such that $\Omega(f-\eta, \lambda)$ is nonvoid. This is seen by 
choosing a point $q=\left(x_{1}, y_{1}, z_{1}\right)$ for which

$$
F(\zeta)=f\left(x_{1}+\frac{1}{2}\left(i y_{1}+z_{1}\right) \zeta+\frac{1}{2}\left(i y_{1}-z_{1}\right) \zeta^{-1}, \zeta\right)
$$

is continuous for all $\zeta$ on $\mathscr{L}_{0}$. The set

$$
\Delta=\left\{x_{1}+\frac{1}{2}\left(i y_{1}+z_{1}\right)+\frac{1}{2}\left(i y_{1}-z_{1}\right) \zeta^{-1} \mid \zeta \in \mathscr{L}_{0}\right\}
$$

is compact and thus has a compact image under $F$ for all $\zeta$ on $\mathscr{L}_{0}$ which we denote by $F\left(\Delta, \mathscr{L}_{0}\right)$. The compactness of $F\left(\Delta, \mathscr{L}_{0}\right)$ guarantees the existence of a vector $\eta_{1}$ such that $f\left(\Delta, \mathscr{L}_{0}\right)-\eta_{1}$ is in $s(\lambda, \omega)$ for some choice of $\omega$. Furthermore, by the analyticity of $f$, a vector $\eta$ can be chosen such that for each point $(x, y, z)$ in a sufficiently small neighborhood of $\left(x_{1}, y_{1}, z_{1}\right)$, the point

$$
G(\zeta)=f\left(x+\frac{1}{2}(i y+z) \zeta+\frac{1}{2}(i y-z) \zeta^{-1}, \zeta\right)-\eta
$$

is in the sector $s(\lambda, \omega)$ for all $\zeta$ on $\mathscr{L}_{0}$.

As applications of this theorem we consider the following corollaries.

COROLlaRY 1. Let $\Psi(x, y, z)$ be a potential generated by $B\left(f, \mathscr{L}_{0}, \boldsymbol{X}\right)$ as in the theorem and let $p$ be a fixed point in $E^{3}$. Let $\Delta$ be a subset of $[0,2 \pi)$, $\gamma$ a real constant and $\eta$ a complex constant such that the family

$$
\{\Gamma(f-\eta, p, \lambda, \delta)\}_{\delta \in \Delta}
$$

has a nonvoid intersection. Then, in $\bigcup_{\delta \in \Delta} \Gamma(f-\eta, p, \lambda, \delta)$,

$$
\Psi(x, y, z,) \neq \xi n\left(\mathscr{L}_{0}, 0\right)
$$

for $\xi=\eta$ or $\xi=\eta+\kappa$ for all $\kappa$ in $\bigcap_{\delta \in \Delta} s(\lambda, \delta+\pi)$.

Proof. We shall consider the case where $\xi=\eta+\kappa$, the other case is proved in a similar manner. Let $(x, y, z)$ be a point in $\bigcup_{\delta \in \Delta} \Gamma(f-\eta, p, \lambda, \delta)$ and consider the integral

$$
\Psi(x, y, z)-\xi n\left(\mathscr{L}_{0}, 0\right)=\frac{1}{2 \pi i} \int_{\mathscr{L}_{0}}(f(\tau, \zeta)-\xi) \zeta^{-1} d \zeta .
$$

We find that, for some $\delta_{0} \in \Delta$,

$$
-\lambda+\delta_{0}<\arg (f(\tau, \zeta)-\eta)<\lambda+\delta_{0}
$$

for each $\zeta$ on $\mathscr{P}_{0}$. For $\kappa \in \bigcap_{\delta \in \Delta} s(\lambda, \delta+\pi),-\kappa \in \bigcap_{\delta \in \Delta} s(\lambda, \delta)$ from which it follows that

$$
-\lambda+\delta_{0}<\arg (f(\tau, \zeta)-\eta-\kappa)<\lambda+\delta_{0}
$$

for all $\zeta$ on $\mathscr{L}_{0}$. The choice of the contour $\mathscr{L}_{0}$, given parametrically by $\zeta=\zeta(t)$ for $0 \leqq t \leqq 1$, implies that

$$
\gamma-\mu<\arg \left(\zeta^{-1}(t) \zeta^{\prime}(t)\right)<\gamma+\mu
$$

for $t$ on $0 \leqq t \leqq 1$. 
By combining inequalities (15) and (16) we find that

$$
\begin{aligned}
\gamma+\delta_{0}-\pi / 2 & <\arg \left\{(f(\tau, \zeta)-\eta-\kappa)\left(\zeta^{-1}(t) \zeta^{\prime}(t)\right)\right\} \\
& <\gamma+\delta_{0}+\pi / 2
\end{aligned}
$$

for each $t$ on $0 \leqq t \leqq 1$. By continuing the reasoning as in the theorem, we find that in $\Gamma\left(\bar{f}-\eta, p, \gamma, \delta_{0}\right), \Psi(x, y, z) \neq(\eta+\kappa) n\left(\mathscr{L}_{0}, 0\right)$ and thus

$$
\Psi(x, y, z) \neq(\eta+\kappa) n\left(\mathscr{L}_{0}, 0\right)
$$

in $\bigcup_{\delta \in \Delta} \Gamma(f-\eta, p, \lambda, \delta)$ as was to be proved.

These techniques may be applied to the Whittaker transform [4, p. 390] to obtain theorems similar to the above results. As an example, we consider Corollary 2 which is proved in a manner similar to that of the theorem.

COROLlaRY 2. Let $g(u, v)$ be an analytic function of $u$ for all $u$ over some region $\Gamma$ in the complex plane where $u=x+i y \cos t+i z \sin t$ and be $a$ continuous function of $v$ for $v$ over $[0,2 \pi]$. Let

$$
\omega(x, y, z)=\frac{1}{2 \pi} \int_{0}^{2 \pi} g(u, t) d t
$$

where $u=x+i y \cos t+i z \sin t$. Then, in $\Omega(g-\eta, \pi / 2)$,

$$
\omega(x, y, z) \neq \eta \text {. }
$$

\section{REFERENCES}

1. Stefan Bergman, Integral operators in the theory of linear partial differential equations, Ergebnisse der Mathematik und ihrer Grenzgebiete, N. F., Heft 23, SpringerVerlag, Berlin, 1961. MR 25 \#5277.

2. Morris Marden, Value distribution of harmonic polynomials in several real variables, Trans. Amer. Math. Soc. 159 (1971), 137-154. MR 43 \#5046.

3. Peter McCoy, Value distribution of axisymmetric potentials, Amer. J. Math. (to appear); Copies available through the Department of Pure and Applied Mathematics, Washington State University, Pullman, Wash.

4. E. T. Whittaker and G. N. Watson, A course of modern analysis. An introduction to the general theory of infinite processes and of analytic functions; with an account of the principal transcendental functions, 4th ed., Cambridge Univ. Press, New York, 1962. MR 31 \#2375.

Department of Pure and Applied Mathematics, Washington State University, PUllman, Washington 99163

Current address: Department of Mathematics, U.S. Naval Academy, Annapolis, Maryland 21402 